\section{Asymmetric inhibition}

Direction-selective ganglion cells (DS cells) in the retina respond to stimuli that move in a particular direction and not to stimuli that move in the opposite (null) direction. This direction sensitivity requires asymmetric inhibitory input from starburst amacrine cells, but how and when this asymmetric wiring is established has remained elusive. Now, two papers published in Nature show that inhibitory input from starburst cells is initially symmetrical but reorganizes and becomes asymmetrical before eye opening, independently of neural activity.

Feller and colleagues studied starburst-DS cell pairs in intact isolated mouse retinas in which excitatory transmission had been blocked. On postnatal day 4 (P4) and P7, depolarization of starburst cells located on the preferred and the null sides ( $\mathrm{P}$ - and $\mathrm{N}$-starburst cells, respectively) of a DS cell induced GABAergic currents of similar magnitude in the DS cell. However, by P14 and through to adulthood, synaptic strength between $\mathrm{N}$-starburst cells and DS cells had increased, whereas that between P-starburst cells and DS cells remained constant.

Roska and colleagues used an optogenetic approach to tackle the same question. In intact mouse retinas they photostimulated starburst cells (expressing channelrhodopsin-2) that surrounded an upward-motion-preferring DS cell, and recorded inhibitory and excitatory currents in the DS cell. In P6 retinas, both excitatory and inhibitory inputs were symmetrically distributed along each DS cell, whereas by $\mathrm{P} 8$ an asymmetric pattern had emerged for inhibitory but not excitatory input: the strongest currents were recorded after photostimulation of N-starburst cells and the weakest currents after photostimulation of P-starburst cells.

Feller and colleagues further showed that the density of contact with DS cells was similar for P- and $\mathrm{N}$-starburst cells, indicating that the differences in synaptic strength were not due to selective adhesion between $\mathrm{N}$-starburst cell processes and DS dendrites. Furthermore, as neither activating nor blocking GABAergic activity in the retina from P6 to P12 affected the direction-sensitive response of DS cells in P14-P15 mice to moving light stimuli, the development of direction sensitivity did not seem to depend on neuronal activity.

These studies suggest that a developmental programme initiates the reorganization of inhibitory input from starburst cells to DS cells between P6 and P8, resulting in a spatially asymmetrical circuit that is key to retinal motion-direction sensitivity.

Leonie Welberg

ORIGINAL RESEARCH PAPERS Wei, W. et al.

Development of asymmetric inhibition underlying direction selectivity in the retina. Nature 5 Dec 2010 (doi: 10.1038/nature09600) | Yonehara, K. et al. Spatially asymmetric reorganization of inhibition establishes a motionsensitive circuit. Nature 19 Dec 2010 (doi:10.1038/nature09711)

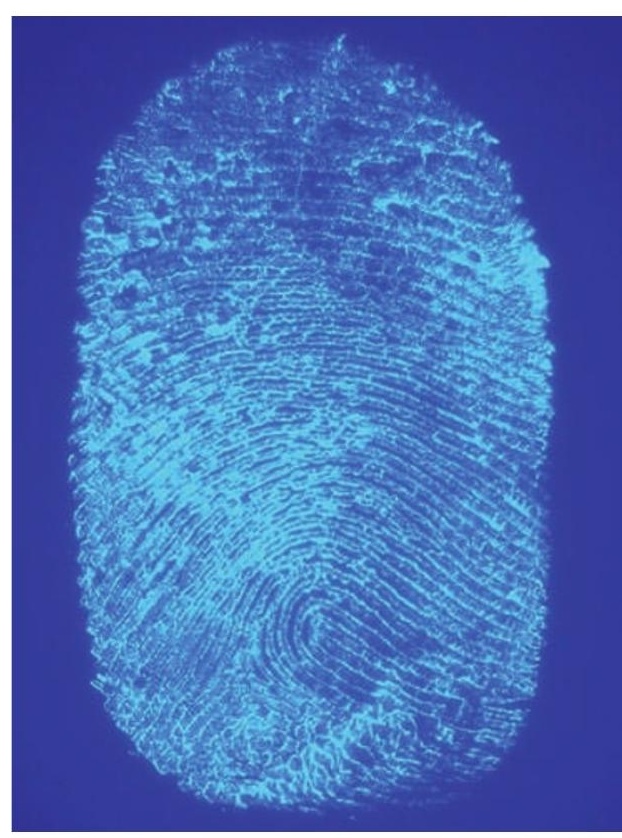

